\title{
Practical Applications of Nonlinear Measurements
}

\author{
Kate A. Remley \\ NIST Electromagnetics Division, Mail Stop 818.02 \\ 325 Broadway \\ Boulder, CO 80305 \\ remley@boulder.nist.gov \\ +1 (303) 497-3652
}

Abstract: We contrast linear, VNA-based measurements, involving ratios of the magnitude and phase of individual frequency components, to measurements of nonlinear devices and circuits, where the relative phase between frequency components and the actual impedance in which a device is embedded are important. The architectures and calibration techniques for several types of instruments designed to satisfy these requirements are presented and compared. Examples of the use of these instruments for measurement and model development of nonlinear circuits are presented.

Keywords: Linear measurements; Measurement-based model; Nonlinear measurements; Nonlinear vector network analyzer; Vector network analyzer.

\section{1(a). Introduction: The Vector Network Analyzer and Linear Measurements}

The vector network analyzer (VNA) was developed to measure ratios of the incident and reflected traveling waves at the input and output ports of an electrical device or circuit at a single frequency (we will use the term "device" to mean a device, circuit, or system). An excitation signal is incident at one port of the device, and the VNA measures the fraction of that signal that is reflected off the port or transmitted through the device. For a linear device, this lets an engineer find many useful parameters, including the scattering, impedance, or admittance parameters of the device and the components in which it is embedded. This allows the engineer to calculate the output voltage and current relative to the input voltage or current. These quantities can, in turn, be used to find the power dissipated in the device and its operating efficiency in the linear regime. For a linear device, changing the amplitude or phase of the excitation will change the complex incident and reflected waves in direct proportion to the excitation, leaving the ratio of the reflected wave to the incident wave unchanged.

Vector network analyzer calibrations are used to correct the measurements of the device to a virtual $50 \Omega$ environment; that is, the calibration provides information about how the device would behave in a $50 \Omega$ environment. Such an impedance transformation is possible only because both the measurement system and the device are linear.

The VNA measures ratios of the incident and reflected traveling waves while stepping through a user-specified range of frequencies one frequency at a time. At each frequency, the traveling waves at the ports of a device-separated into their incident and reflected components by use of directional couplers-are downconverted by a

\footnotetext{
${ }^{*}$ Publication of the U.S. government, not subject to copyright in the United States.
} 
mixer and sampled, as shown in the block diagram of Figure 1. The VNA reports measurements of the reflected or transmitted waves relative to the input signal amplitude and phase. Because the VNA has been developed with linear device measurements in mind, there is no need to know the absolute value of the measured quantities; only ratios are required.

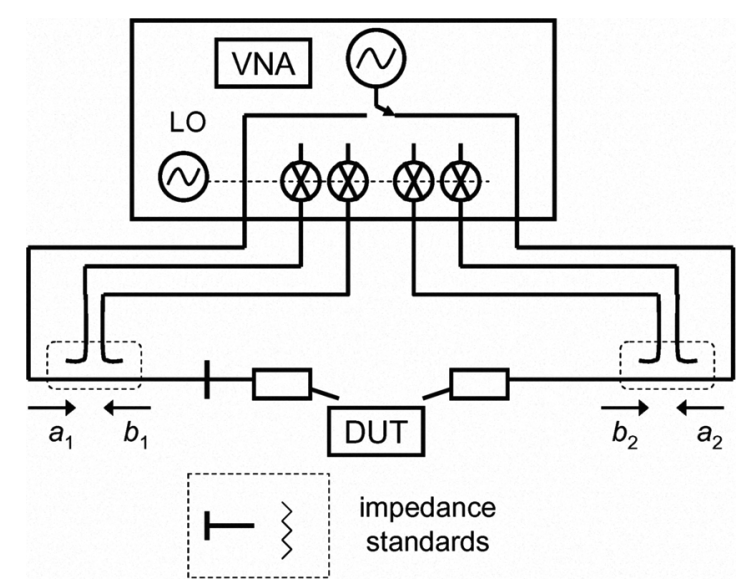

Figure 1: Block diagram of a vector network analyzer. The source excites ports one and two sequentially by switching between the two. The undriven port is terminated in a $50 \Omega$ impedance. Directional couplers are used to measure the forward $\left(a_{i}\right)$ and reflected $\left(b_{i}\right)$ traveling waves. For a two-port VNA, $i=1,2$. The measured signal is sampled and displayed as a ratio. Calibration is carried out by connecting impedance standards at a reference plane, denoted by a vertical line on the port 1 side in the figure. In practice, reference planes exist at both ports.

One common telecommunications application of the VNA is to measure the small-signal gain of an amplifier when it is in a $50 \Omega$ environment. In this application, the VNA is used to characterize an amplifier when it is operating in its "weakly nonlinear" regime. In the weakly nonlinear regime, distortion products are created at a negligibly low level, and the transistor output response increases approximately linearly in proportion to the increase in the excitation level. Figure 2 shows a measurement of the output power of a transistor at the fundamental and harmonic frequencies as a function of the input power [1]. For the measurements shown in Figure 2, the device response for input power levels below around $-15 \mathrm{dBm}$ to $-20 \mathrm{dBm}$ could be considered as weakly nonlinear. For a $-20 \mathrm{dBm}$ input power level, the third-order intermodulation product under two-tone excitation is almost $50 \mathrm{~dB}$ lower than the output signal at the fundamental. Use of a VNA to measure transistor response in this region is common, even though VNAs cannot generally measure the distortion products. 


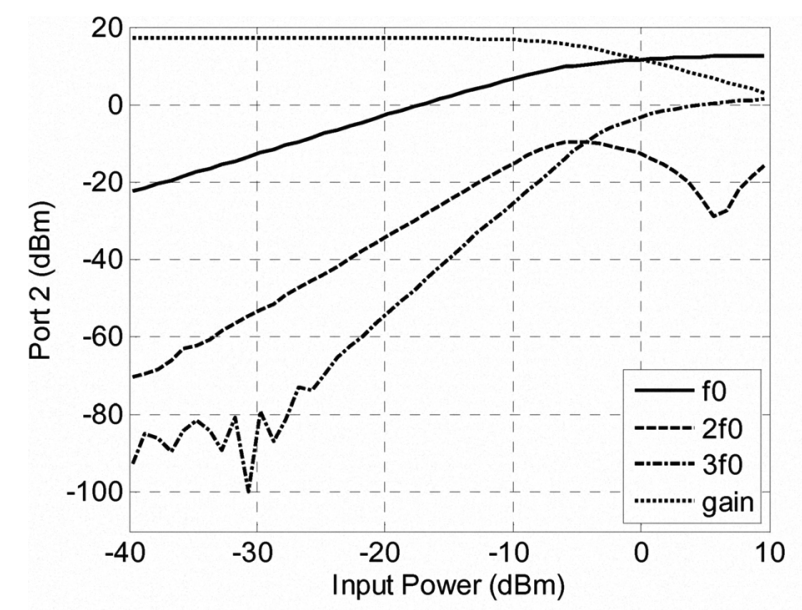

(a)

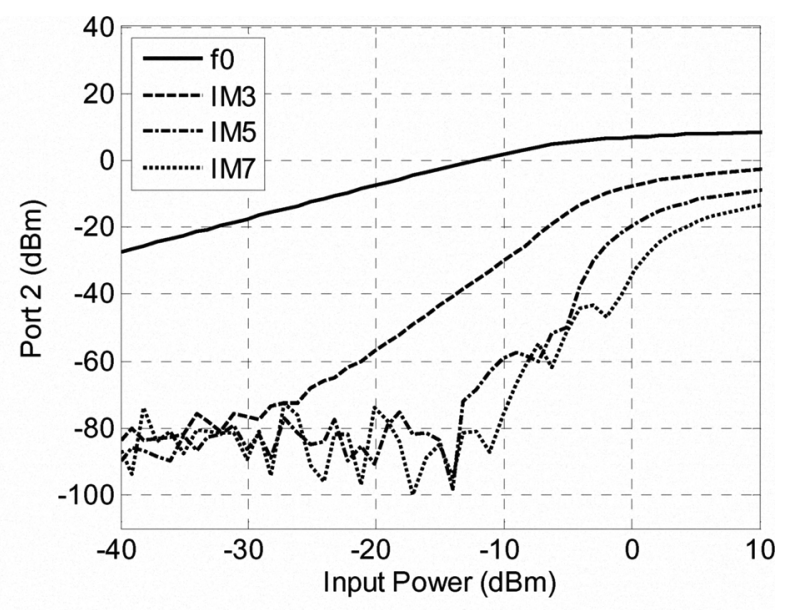

(b)

Figure 2: Measurements of the output power as a function of input power for a $0.12 \mu \mathrm{m}$ CMOS transistor, illustrating the evolution of (a) harmonic and (b) intermodulation distortion. The measurements were made with an instrument capable of measuring nonlinear phenomena under large-signal conditions (after [1]).

\section{1(b) Measurements of Nonlinear Effects}

Nonlinear circuits generate frequency components at frequencies other than that of the excitation. For some applications, such as mixer circuits, this is desirable. In a mixer, we apply an RF signal, the local oscillator (LO), whose amplitude is high enough to place a nonlinear element such as a diode, into its nonlinear operating state. If a smaller signal-one that is small enough not to perturb the large-signal condition-is applied at a frequency offset from the LO, the nonlinear device creates copies of the small signal around the harmonics of the local oscillator frequency. These are the wellknown "RF" and "image" frequencies. The RF and image frequencies can be measured by the VNA since they are linearly transformed small-signal copies of the original signal.

In other cases, nonlinearity is not a desirable phenomenon, because the creation of additional frequency components may impair the performance of a device or system. For example, in cellular telephone applications, it is desirable to transmit as much data as possible while using the battery's power as efficiently as possible. Power amplifiers in cell phone applications are biased as close to their compression levels as possible so that the battery is not used to maintain a bias in excess of that needed for amplification. Compression can distort the transmitted signal and cause generation of frequency components outside the allocated channel.

Some digital modulation transmission formats use signals with high peak-toaverage power ratios. To transmit these peaks without distortion, it is necessary either to bias the amplifier in an inefficient Class A mode, which ensures linear transmission, or to use creative methods to retain efficient transmission such as predistortion, where an amplifier is allowed to compress and the distortion is corrected in pre- and postprocessing. Alternatively, creative transmission schemes such as the Doherty amplifier may be used, where the signal's peaks are amplified separately. To facilitate design and 
verify the performance of these methods, it is useful to be able to measure both the magnitudes and relative phases of the distortion products.

Because the VNA generates a single-frequency excitation signal and measures at that same frequency, it cannot be use to measure harmonics and distortion products generated at other frequencies. Also, from Figure 2, it is clear that in order to understand at what power level distortion occurs, we need to know the absolute value of the signal incident on the nonlinear device. Ratios are no longer sufficient.

Some VNA measurement methods have been proposed to help get around this issue. For example, by connecting a power meter to the input or output port of a VNA, we can measure the magnitude of the distortion products [2, 3], as shown in Figure 3. Alternatively, a spectrum analyzer can be used to measure the level of the distortion products when the nonlinear device is excited with an external source. The excitation and the measurement do not have to occur at the same frequencies. Use of a spectrum analyzer in conjunction with an impedance calibration forms the basis for many load-pull systems. In these systems, tuners are used to modify the output-port terminating impedance. Distortion products are measured in order to determine the best impedance match for (for example) an amplifier circuit.

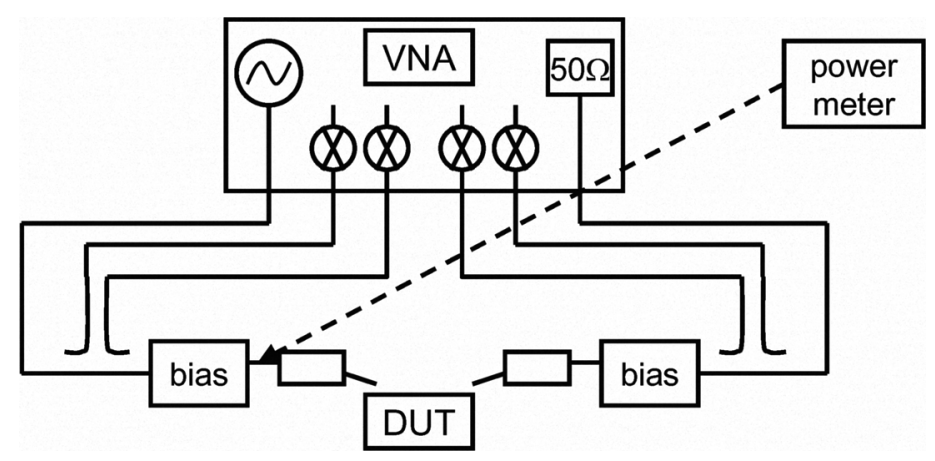

Figure 3: A power meter can be connected to a VNA test set to measure the power in distortion products that are generated at frequencies other than the excitation frequency. The power meter can also be used to calibrate the fundamental power incident on the device (after [2]).

However, power meters and spectrum analyzers do not provide a key piece of information about nonlinear behavior: the relative phase between the frequency components. This information is important if we wish to study the phase of the distortion products, reconstruct a time-domain waveform, or demodulate a digitally modulated signal. As an example of the necessity of accurate phase information for waveform reconstruction, Figure 4(a) shows a $1 \mathrm{GHz}$ square wave measured on a digital sampling oscilloscope (dotted line) and a large-signal network analyzer (LSNA) whose phase response is either calibrated (dashed line) or left uncalibrated (solid line). The effect of leaving the phase uncalibrated is that the measured waveform is radically different from the one that was present at the output of the signal generator. As shown in Figure 4(b), the magnitude of the spectral content is the same for all of the measurements. 


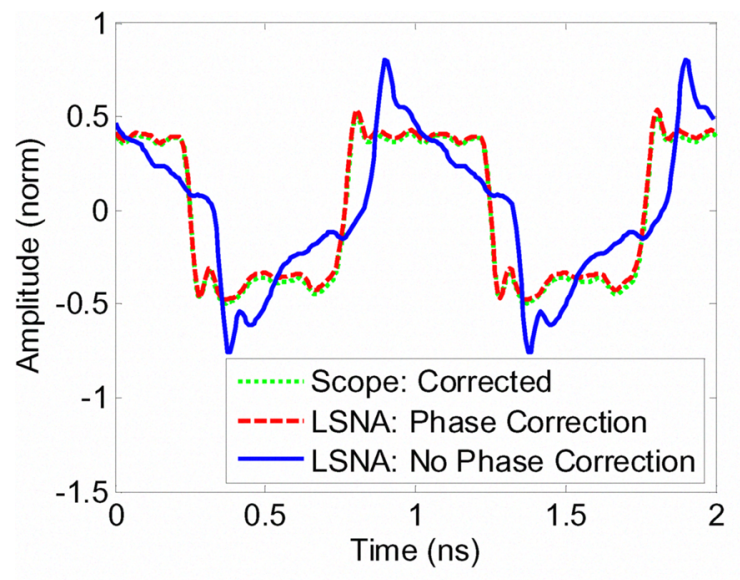

(a)

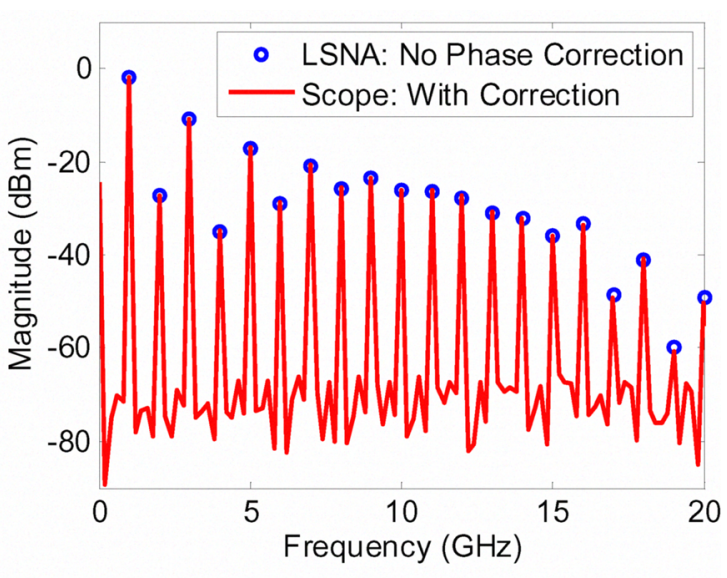

(b)

Figure 4: Measurement of a $1 \mathrm{GHz}$ square wave. (a) Time domain representation comparing LSNA measurements made with and without phase calibration. The corrected measurements nearly overlay on this scale. In (b), we see that the spectrum is not affected by the lack of a phase calibration, even though (a) clearly shows higher peak waveform values (after [4]).

A second application where it is important to measure the phase between frequency components is in the measurement of peak power in a modulated signal. In telecommunications applications, if the peak is high, it can cause out-of-band interference and/or damage the components in the system. Figure 5 shows an example of two modulated signals having the same transmitted power and frequency components with the same amplitudes but different relative phases. Obviously, one of the signals has a much higher peak-to-average power ratio (sometimes called crest factor).

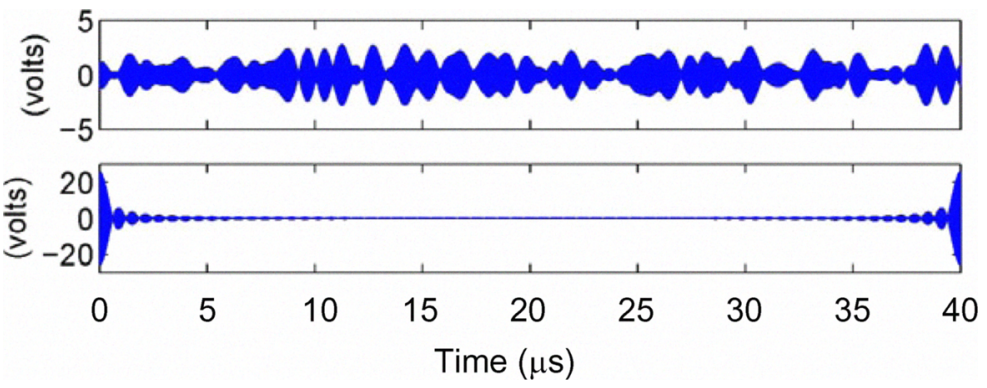

Figure 5: Two time waveforms having the same total power and the same 65-tone amplitude spectrum. However, the different relative phase relationships between frequency components yield very different peak-to-average power ratios. Note the different scale on the $y$ axes (from [5]).

The impedance in which a device is embedded takes on a new and important role in the measurement of nonlinear devices as well. As was just discussed, nonlinear device operation depends on the magnitude and phase of the signals present at its input and output ports. As a result, the actual impedance in which the device is 
embedded will affect the operation of the nonlinear device. For example, whether an amplifier is terminated in a $50 \Omega$ load or some other value can significantly affect its efficiency. Furthermore, the input and output impedances of an amplifier are often a function of the input signal level. Simply transforming the embedding impedance into a virtual $50 \Omega$ environment when an amplifier is out of the weakly nonlinear regime does not provide enough information for successful characterization of the nonlinear device. And, because frequency conversion is going on under nonlinear operating conditions, the impedances at both the harmonics and at baseband may affect the device operation as well.

The VNA was designed with a different set of operating conditions in mind that are often found in nonlinear applications: they measure relative quantities at single frequencies and, rather than providing explicit knowledge of embedding impedances, they transform them to a reference value. These features work well for linear applications but usually do not provide enough information in nonlinear applications. Instruments with different architectures have been developed specifically to capture the absolute magnitude and phase information, and often the embedding impedance, for nonlinear applications.

\section{2(a). The Essential Components of Nonlinear Measurement Systems}

The defining elements of nonlinear measurements were outlined above. They include the ability to measure the absolute amplitude of signals at the ports of a device as well as the ability to measure the relative phase between frequency components, especially those at frequencies other than the excitation frequency. In many cases, the ability to measure the impedance at the fundamental and harmonic frequencies is also important. The measured magnitude and phase can be used to derive the impedance if directional couplers are used.

Instruments used to measure nonlinear devices and circuits (often called "nonlinear measurement systems" even though the instruments are not themselves nonlinear) use a variety of techniques to maintain or reconstruct the phase relationships between frequency components. If the instrument is designed to measure at multiple ports, the phase relationships between signals measured at each port are maintained as well, just as they are in a VNA. To determine the true impedance present at the port, the incident and reflected waves are recorded individually, rather than as a ratio, as is measured by most VNAs.

Current commercially available instruments that can measure the relative phase between frequency components include oscilloscopes (both real-time and sampling), vector signal analyzers (VSAs), real-time signal analyzers (RTSAs), large-signal network analyzers (LSNAs), and nonlinear vector network analyzers (NVNAs) based on VNAs. There are a host of systems developed by research institutions and labs as well. These instruments use various methods to acquire a signal so that the phase relationships between frequency components are maintained. In addition to providing knowledge of absolute signal levels, this is one of the key distinguishing factors between measurement systems used for nonlinear applications and VNAs.

One instrument that uses elements of real-time sampling, where the time-domain waveform is sampled sequentially, is the vector signal analyzer. This instrument was 
designed to allow engineers to study distortion introduced into digitally modulated bandpass RF signals. In order to demodulate the signals, the phase relationships between frequency components are maintained by the instrument, qualifying it as a "nonlinear measurement system." As shown in the block diagram of Figure 6, the time waveform acquired by the VSA is first bandpass filtered and downconverted to an intermediate frequency. It is then digitized by use of a fast sampler. The digitized waveform is separated into its in-phase and quadrature components and is then transformed to the frequency domain. In many respects, the VSA is like a real-time sampling oscilloscope, but higher dynamic range and frequency resolution are obtained by limiting the acquisition bandwidth.

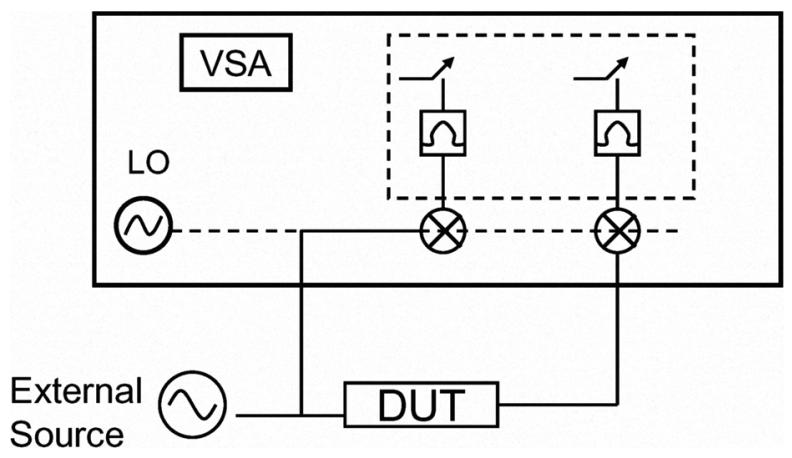

Figure 6: Block diagram of a vector-signal analyzer. The downconverted, bandpass-filtered signal is sampled in real time by high-speed analog-to-digital converters.

As mentioned above, several types of nonlinear measurement systems have been developed to provide impedance information in addition to absolute magnitude and phase, including [6-19]. These instruments use impedance standards and directional couplers to separate the incident and reflected waves and transform the measured impedances to the reference plane of the DUT. Currently available instruments utilize periodic excitation so that multi-harmonic signals can be sampled and reconstructed. We describe some of the most common architectures below, but because this is an active area of research, new architectures are being developed at a rapid pace.

Sampler-based LSNA systems such as those described in [6 - 10], shown in Figure 7(a), use a technique called harmonic mixing or "sampling downconversion" [7] to acquire the fundamental and harmonic frequency components at the input and output ports of a device simultaneously, retaining the phase information between them. In sampling downconversion, a local oscillator (LO) operating at a relatively low frequency (for example, around $20 \mathrm{MHz}$ ) is used to trigger the samplers. The exact frequency at which the LO operates is chosen such that the difference between selected LO harmonics and the harmonics of the RF frequencies lie within the IF bandwidth of the system. Because the chosen harmonics of the LO and the RF signals are slightly offset in frequency, the samplers step through the repeating RF waveform. Harmonically related frequency components of the RF signal are sampled and downconverted to baseband, where they are bandpass filtered. 


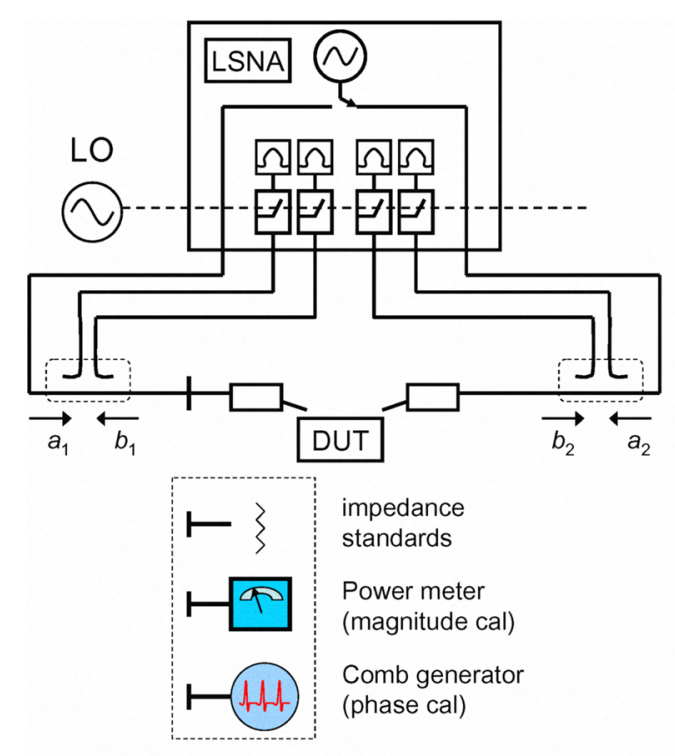

(a)

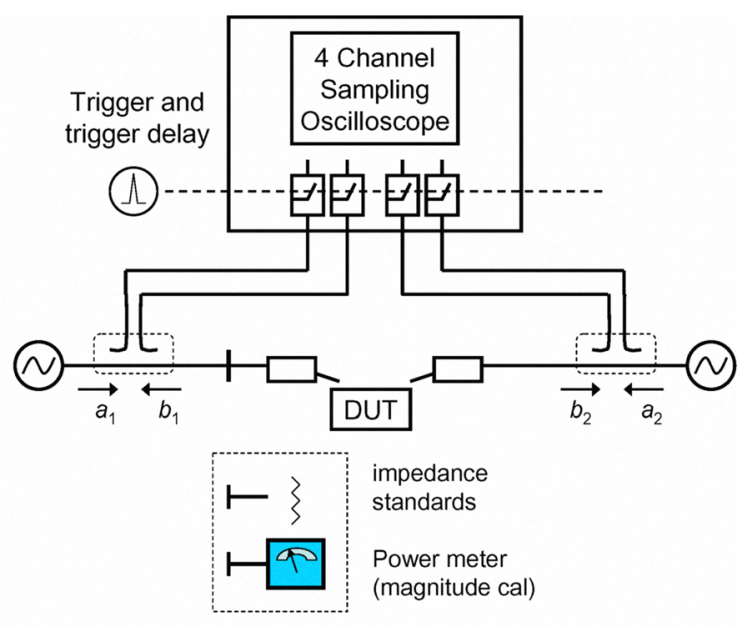

(b)

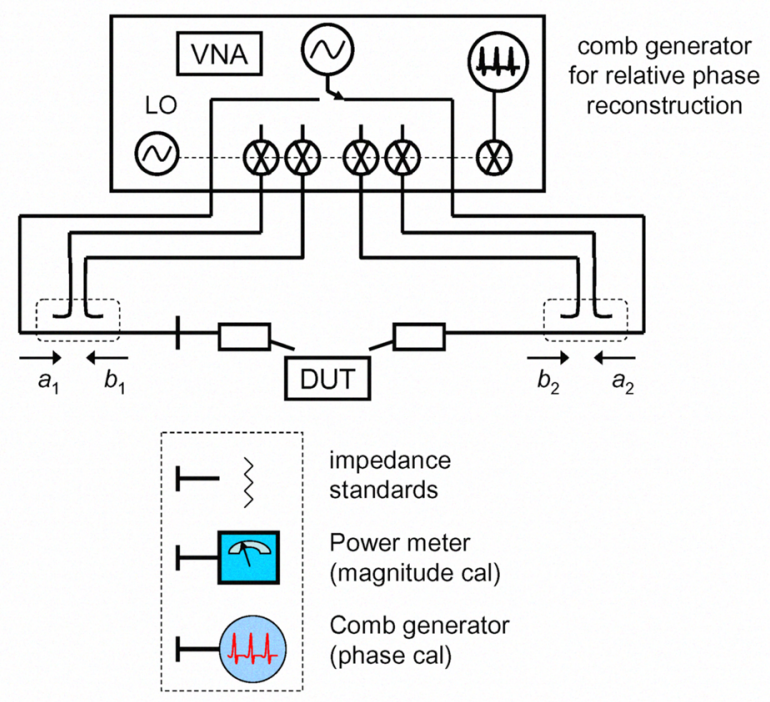

(c)

Figure 7: Three architectures for nonlinear measurement systems. (a) In the system of [6], sampling downconversion is used to measure the relative phases between the fundamental and harmonics. (b) In the system of [12], a multi-channel equivalent-time sampling oscilloscope is used to acquire the waveforms at the input and output ports of a device, retaining the magnitude and phase information. (c) The system of [15] is similar to a VNA, with the addition of a comb generator applied to one of the receivers to reconstruct the relative phases of the measured signal.

Waveform measurement systems such as those described in [11, 12], shown in Figure 7(b), have a test set similar to the VNA, but use sampling oscilloscopes instead of samplers or mixers. Use of an oscilloscope provides a measurement whose speed is 
fixed, regardless of the complexity of the signal. These instruments measure over a broad bandwidth, not limited to acquisition around harmonics of the excitation, with a corresponding reduction in dynamic range when compared to the system shown in Figure 7(a). The measurement accuracy of the oscilloscope can be affected by both time-base distortion and jitter, although these can be corrected by use of oscilloscopes having additional channels with the methods described in, for example, [20].

A different architecture, based on a VNA with the addition of the power and phase calibrations, is described in [13 - 15]. The system of [15] consists of a VNA with an extra receiver to measure the output of a comb generator. In this way, the relative phase of each frequency component can be reconstructed as it is measured (see Figure $7(\mathrm{c})$ ). This system retains the high dynamic range of a VNA, as do the earlier VNAbased systems described in [13,14], which used diode-based comb generators as phase references. As with the instruments described above, the excitation must be periodic, so that the signals can be sampled and reconstructed.

\section{2(b) Calibration Techniques}

Most nonlinear measurement systems, including those shown in Figures 7(a) (c), use standard VNA calibrations to transform the measurement reference plane to the DUT. These impedance transformations are applied across the linear components in the system, just as for a VNA.

To calibrate the absolute magnitude of the signals measured by systems such as those shown in Figures 7(a) and 7(c), a power meter is used as an additional calibration standard (traceable to a national measurement institute (NMI)). The magnitude response of oscilloscope-based instruments, such as the one shown in Figure 7(b), can be calibrated by use of a swept-sine approach, where time-domain waveforms of singlefrequency signals are acquired and calibrated with a power meter at multiple frequencies. Other instruments, such as the VSA, are calibrated periodically by the manufacturer.

To calibrate measurements of the relative phase relationships between frequency components, the systems shown in Figures 7(a) and 7(c) use a phase reference such as a comb generator. During phase calibration, the signal source is eliminated from port one of the system and the output of the comb generator is measured instead. By comparing the measured phases of the comb generator to the phases measured at the factory, calibration coefficients are determined and applied to measurements. A calibrated sampling oscilloscope is used to provide the factorymeasured phase reference. NMls including NIST, NPL, and PTB have developed sampling oscilloscope calibrations that are traceable to fundamental physical units based on electro-optic sampling. The NIST procedure includes impedance mismatch correction and is thus useful for calibrating the comb generators used to calibrate NVNAs. The NIST calibration procedure $[21,22]$ is outlined in Figure 8. 

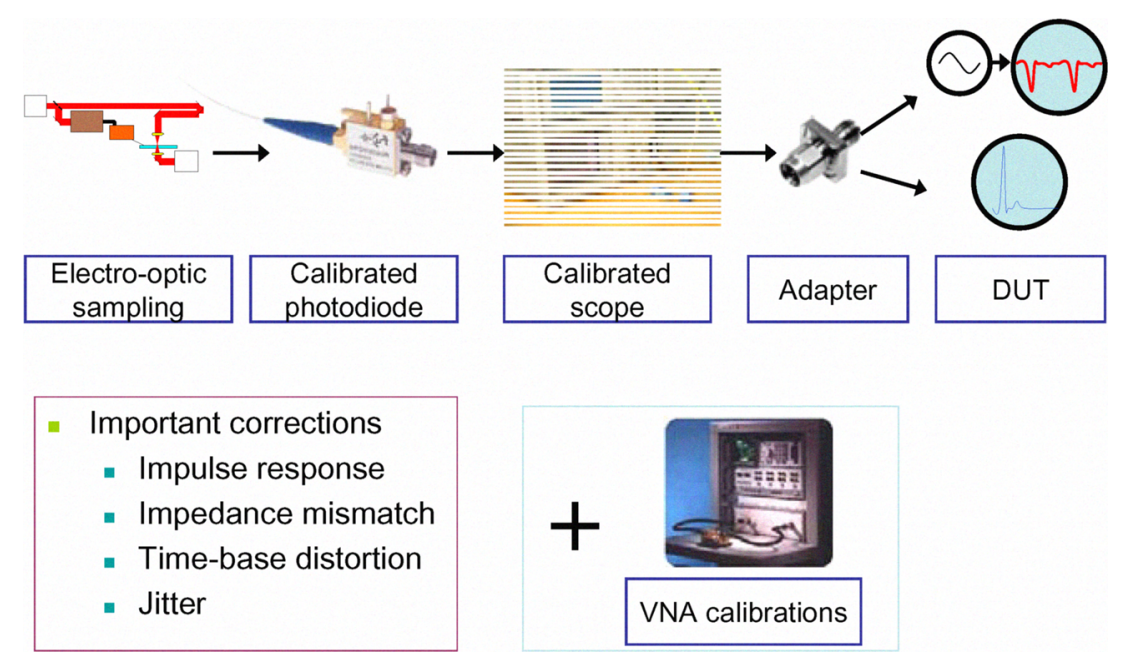

Figure 8: NIST calibration procedure for a sampling oscilloscope, showing the entire traceability chain.

\section{3(a) Example 1: Effects of Embedding Impedance on Nonlinear Device Measurements}

To illustrate some practical applications of nonlinear measurements, we first show how the impedance in which a nonlinear device is embedded can affect measurements of harmonic distortion. In Figure 9(a), we show a power sweep of the same transistor presented in Figure 2, a $0.12 \mu \mathrm{m}$ CMOS device [1]. Similar devices were measured by use of a large-signal network analyzer at Lab 1 and by use of a loadpull system at Lab 2.

We see that the measurement of the second harmonic shown by the thin dotted line (labeled "Lab 2 Original") is several decibels lower than that given by the solid thin lines or the symbols. The reason for this difference is shown in Figure 9(b), where we see that the second harmonic impedance for the original load-pull measurement is quite different from the nearly $50 \Omega$ impedance measured by the Lab 1 instrument and by the load pull system labeled "Matched" in Figure 9(a). The LSNA used by Lab 1 was calibrated at the fundamental and harmonics and was used to troubleshoot the Lab 2 measurements.

The impedance that measurement systems (both receivers and sources) present to the DUT at the baseband can also affect the level of intermodulation distortion measured at RF, as can harmonic distortion generated by the sources. This has been discussed by many researchers and is one of the reasons why comparing measurements made on different nonlinear measurement systems is difficult: the impedances at RF, at the harmonics, and at baseband all affect the operation of the nonlinear device. It is difficult to control all of these impedances simultaneously from instrument to instrument. Approaches to circumvent this are proposed in [23, 24], for example. 

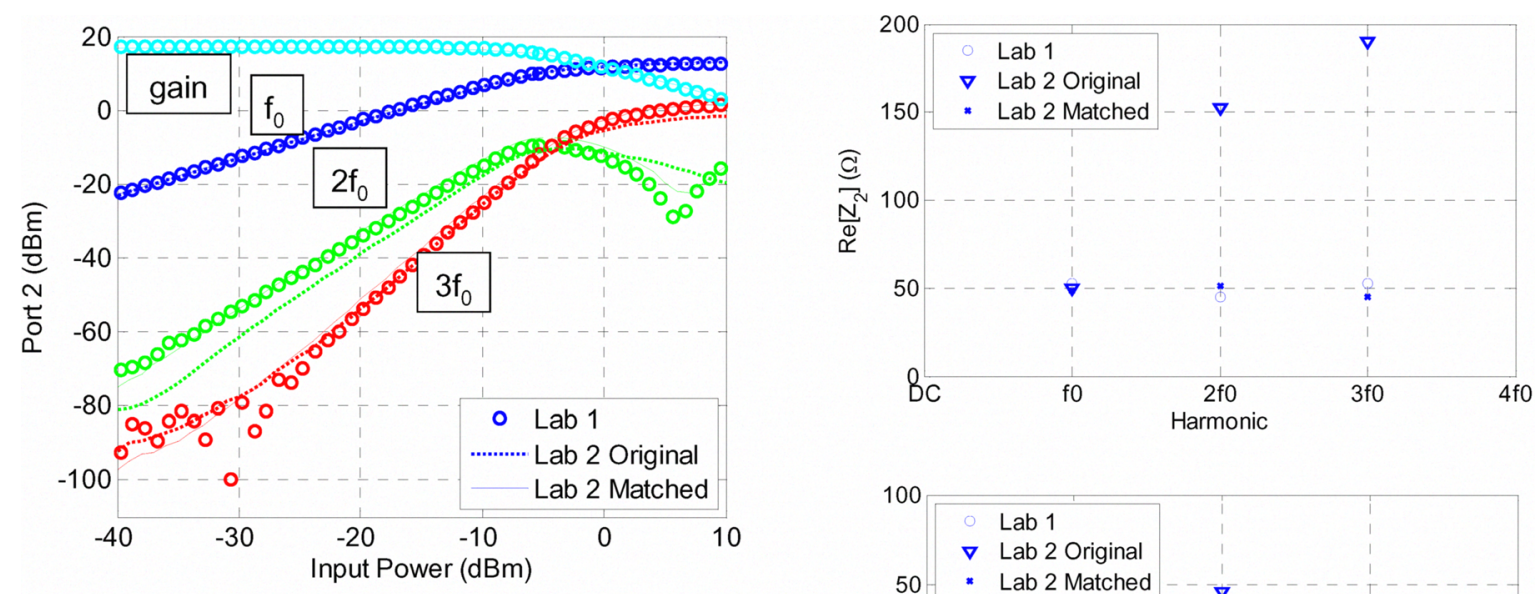

(a)

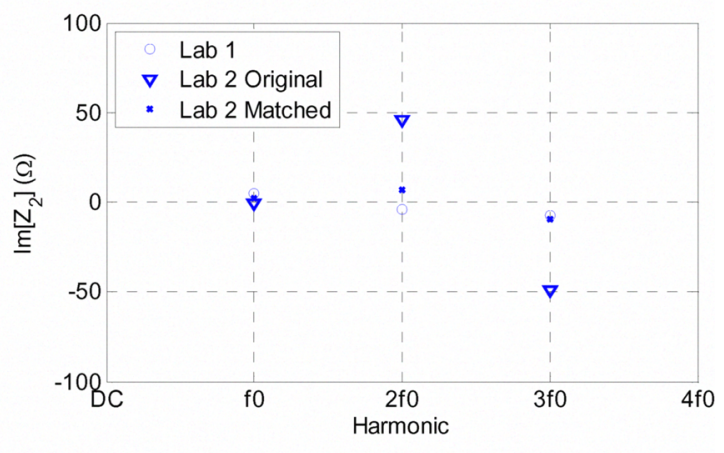

(b)

Figure 9: Measurements of the output power as a function of the input power for a $0.12 \mu \mathrm{m}$ CMOS device [1]. (a) The measured values of the second harmonic distortion product, labeled $2 f_{0}$, are different from each other. (b) The source of the difference was the terminating impedance presented by the load-pull system at the second harmonic.

\section{3(b) Example 2: Measurement-Based Models}

One common application of measurements of nonlinear circuits is in the development of measurement-based models. Models of transistors in their large-signal operating state can be used to predict the performance of the transistor when it is placed in a circuit.

Such models can be generated by extracting key device operating parameters from measurements. For example, in [1], a "compact" model (that is, one based on an equivalent circuit of the device) was developed from DC current/voltage measurements of the device and by modifying circuit parameters to match the measured harmonic and intermodulation distortion when the model was placed in the same impedance environment in which it was measured.

Black-box models, which are based on the measured input-to-output device performance, can be extracted from large-signal measurements in a number of ways (see for example [23 - 25], to name but a few). Often these models specify the response of the modeled element to excitation at key frequencies of operation. To verify the performance of these models, we can place the black box model in a circuit simulator and excite the model by the measured incident waves from both ports one and two using the procedure diagramed in Figure 10(a) [26]. By comparing the simulated 
reflected waves to the measured reflected waves, we can see how well the model replicates the device response in a given impedance environment. An example of this is shown in Figure 10(b), where we see that the simulated and measured $b_{2}$ waves match well at the fundamental (to within $1 \mathrm{~dB}$ ), but less so at the second harmonic (to within 10 $\mathrm{dB}$ for the higher-order intermodulation distortion products).

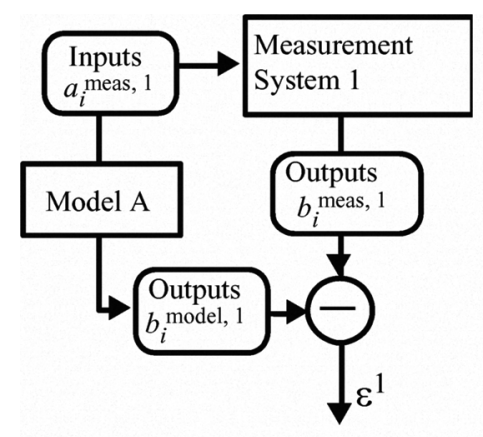

(a)
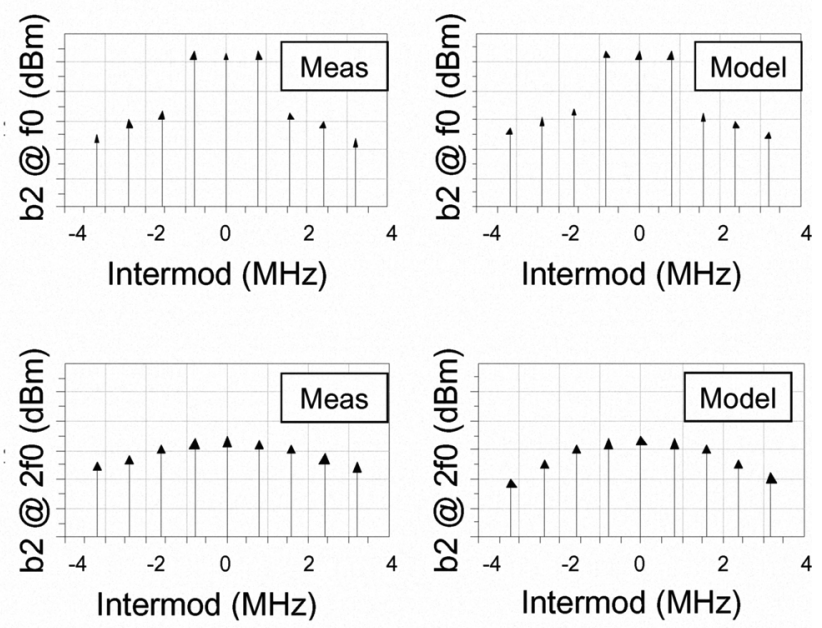

(b)

Figure 10: (a) Flow chart showing a method for verifying Model A by comparing the model's reflected $\left(b_{1}\right)$ and transmitted $\left(b_{2}\right)$ waves to the measured $b_{1}$ and $b_{2}$ waves when both the model and the DUT are excited by the same incident $a_{1}$ and $a_{2}$ waves (b) Left graphs are extracted directly from measurements and right graphs are from the model. The $y$-axis on all graphs ranges from $-100 \mathrm{dBm}$ to $+20 \mathrm{dBm}$ with $10 \mathrm{~dB}$ steps between the major and minor ticks. At the fundamental (top graphs), the measurement and the model agree to within $1 \mathrm{~dB}$. At the second harmonic, agreement is somewhat less good, with differences up to $10 \mathrm{~dB}$ between the measured and modeled cases for the higher-order intermodulation distortion products (from [26]).

\section{Conclusion}

Vector network analyzers cannot generally be used to study devices and circuits under nonlinear operating conditions because they excite and measure at only one frequency, while nonlinear devices generate additional frequency components. The VNA is also unable to measure the absolute magnitude and phase of the waves incident on and reflected from the device under test. This is necessary to measure the distortion arising from nonlinear devices in both small- and large-signal operating conditions.

A number of instrument architectures have been developed for the purpose of nonlinear vector measurement, with more being invented all the time. Power meters are generally used to ensure that instruments are measuring the magnitudes correctly. To measure the phase relationships between frequency components, most instrument architectures either rely on time-domain waveform measurements or use comb generators as phase references.

Telecommunications applications continue to become increasingly complex in the quest for increasingly efficient spectral usage, with wider modulation bandwidths, higher-order modulation schemes, and higher carrier frequencies. With this trend, the 
need to accurately measure nonlinear effects is also increasing. Nonlinear measurement systems will play an increasingly important role in measurement of key parameters such as complex distortion, waveform purity, and device impedance.

\section{Acknowledgement}

We are grateful to Dylan Williams and Paul Hale of NIST for collaborations on oscilloscope measurement methods, including the creation of Figure 8 and the work in [4], and to Dominique Schreurs and Maciej Myslinski of the K.U. Leuven for collaborations on nonlinear device measurements and models, including the work presented in Example 2. Daniel Gunyan of Agilent Technologies, Johannes Benedickt of Cardiff University, and Frans Verbeyst of NMDG Engineering also provided helpful comments on the manuscript.

\section{References}

[1] K.A. Remley, J. Gering, S. Sweeney, C.M. Olsen, C. Xie, D. Walker, T. McKay, J. Pekarik, "Inter-Laboratory Comparison of CMOS Distortion Measurements," 69th ARFTG Conf. Dig., Honolulu, HI, pp. 14-26, June. 2003.

[2] D.C. Kerr, J.M. Gering, T.G. McKay, M.S. Carroll, C.R. Neve, and J.-P. Raskin, "Identification of RF harmonic distortion on Si substrates and its reduction using a traprich layer," IEEE Radio and Wireless Conf., pp. 151-154, Jan. 2008.

[3] J. Dunsmore, "Novel method for vector mixer characterization and mixer test system vector error correction," IEEE MTT-S Int. Microwave Symp. Dig., vol. 3, pp. 1833-1836, Jun. 2002.

[4] K.A. Remley, P.D Hale, and D.F. Williams, "Absolute magnitude and phase calibrations," in RF and Microwave Circuits, Measurements, and Modeling, M. Golio and J. Golio, eds., Boca Raton, FL: CRC Press, Taylor and Francis Group, 2008.

[5] K.A. Remley, "Multisine excitation for ACPR measurements," IEEE MTT-S Int. Microwave Symp. Dig., pp. 2141-2144, June 2003.

[6] T. Van den Broeck, J. Verspecht, "Calibrated vectorial nonlinear-network analyzers," IEEE MTT-S Int. Microwave Symp. Dig., pp.1069-1072, May 1994.

[7] J. Verspecht, "The return of the sampling frequency convertor," $62^{\text {nd }}$ ARFTG Conf. Dig, Boulder, CO, pp. 155-164, Dec. 4-5, 2003.

[8] J. Verspecht, "Large-signal network analysis," IEEE Microwave Magazine, vol. 6, no. 4, pp. 82-92, Dec. 2005.

[9] W. Van Moer and Y. Rolain, "A large-signal network analyzer: Why is it needed?," IEEE Microwave Magazine, vol. 7, no. 6, pp. 46-62, Dec. 2006. 
[10] M. El Yaagoubi, G. Neveux, D. Barataud, T. Reveyrand, J.-M. Nebus, F. Verbeyst, F. Gizard, and J. Puech, "Time-domain calibrated measurements of wideband multisines using a large-signal network analyzer," IEEE Trans. Microwave Theory Tech., vol. 56, no. 5, pp. 1180-1192, May 2008.

[11] M. Sipilä, K. Lehtinen, and V. Porra, "High-frequency periodic time-domain waveform measurement system," IEEE Trans. Microwave Theory Tech., vol. 36, no. 10, pp. 1397-1405, Oct. 1988.

[12] T. Williams, J. Benedikt, and P.J. Tasker, "Fully functional 'real time' non-linear device characterization system incorporating active load control," $36^{\text {th }}$ European Microwave Conf. Dig., pp. 1610-1613, Sept. 2006.

[13] U. Lott, "Measurement of Magnitude and Phase of Harmonics Generated in Nonlinear Microwave Two-Ports", IEEE Trans. Microwave Theory Tech., vol. 37, no. 10, pp. 1506-1511, Oct. 1989.

[14] D. Barataud, et al., "Measurements of time-domain voltage/current waveforms at $\mathrm{RF}$ and microwave frequencies, based on the use of a vector network analyzer for the characterization of nonlinear devices - application to high-efficiency power amplifiers and frequency multipliers optimization," IEEE Trans. Instrum. Meas, vol. 47, no. 5, pp.1259-1264, Oct. 1998.

[15] P. Blockley, D. Gunyan, and J.B. Scott, "Mixer-based, vector-corrected, vector signal/network analyzer offering $300 \mathrm{kHz}-20 \mathrm{GHz}$ bandwidth and traceable phase response," IEEE MTT-S Int. Microwave Symp. Dig, pp. 1497-1500., June 2005.

[16] G. Kompa and F. van Raay, "Error-corrected, large-signal waveform measurement system combining network analyzer and sampling oscilloscope capabilities," IEEE Trans. Microwave Theory Tech., vol. 38, no. 4, pp. 358-365, Apr. 1990.

[17] F. van Raay and G. Kompa, "A new on-wafer large-signal waveform measurement system with $40 \mathrm{GHz}$ harmonic bandwidth," 1992 IEEE MTT-S Int. Microwave Symp. Dig., vol. 3, pp. 1435-1438, June 1992.

[18] M. Demmler, P.J. Tasker, and M. Schlechtweg, "On-wafer large signal power, Sparameter and waveform measurement system," Conf. Rec. Third Int. Workshop Integrated Nonlinear Microwave Millimeterwave Circuits, Duisburg, Germany, pp. 153158, Oct. 1994.

[19] J.B. Scott, P.S. Blockley, and A.E. Parker, "A new instrument architecture for millimetre-wave time-domain signal analysis," $63^{\text {rd }}$ ARFTG Conf. Dig, Fort Worth, TX, pp. 47-51, June 2004. 
[20] P. D. Hale, C. M. Wang, D. F. Williams, K. A. Remley, and J. Wepman, "Compensation of random and systematic timing errors in sampling oscilloscopes," IEEE Trans. Instrum. Meas., vol. 55, no. 6, pp. 2146-2154, Dec. 2006.

[21] D. F. Williams, A. Lewandowski, T. S. Clement, C. M. Wang, P. D. Hale, J. M. Morgan, D. Keenan, and A. Dienstfrey, "Covariance-Based Uncertainty Analysis of the NIST Electro-optic Sampling System," IEEE Trans. Microwave Theory Tech., vol. 54, no. 1, pp. 481-491, Jan. 2006.

[22] T. S. Clement, P. D. Hale, D. F. Williams, C. M. Wang, A. Dienstfrey, and D. A. Keenan, "Calibration of sampling oscilloscopes with high-speed photodiodes," IEEE Trans. Microwave Theory Tech. , vol. 54, no. 8, pp. 3173-3181, Aug. 2006.

[23] D.E. Root, J. Verspecht, D. Sharrit, J. Wood, A. Cognata, "Broad-band polyharmonic distortion (PHD) behavioral models from fast automated simulations and large-signal vectorial network measurements," IEEE Trans. Microwave Theory Tech., vol. 53, no. 11, pp. 3656-3664, Nov. 2005.

[24] F. Verbeyst and M. Vanden Bossche, "Speed up power amplifier design by fast source-pull, real-time load-pull and accurate measurement-based behavioural models," Microwave Engin. Europe, pp. 24-30, Nov. 2005.

[25] D. Schreurs, K. A. Remley, M. Myslinski, R. Vandersmissen, "State-space modelling of slow-memory effects based on multisine vector measurements," 62nd ARFTG Conf. Dig., Boulder, CO, pp. 81-88, Dec. 4-5, 2003.

[26] K.A. Remley, D.C. DeGroot, J. A. Jargon, and K. C. Gupta, "A method to compare vector nonlinear network analyzers," IEEE MTT-S Int. Microwave Symp. Dig., pp. 16671670, May 2001. 